\title{
Suicídio e povos indígenas brasileiros: revisão sistemática
}

\author{
Ronaldo Santhiago Bonfim de Souza, ${ }^{1}$ Júlia Costa de Oliveira, ${ }^{2}$ Juliana Alvares-Teodoro ${ }^{3}$ e Maycoln \\ Leôni Martins Teodoro'
}

Como citar Souza RSB, Oliveira JC, Alvares-Teodoro J, Teodoro MLM. Suicídio e povos indígenas brasileiros: revisão sistemática. Rev Panam Salud Publica. 2020;44:e58. https://doi.org/10.26633/RPSP.2020.58

RESUMO

\begin{abstract}
Objetivo. Descrever a frequência, as características e os fatores que contribuem para o suicídio em povos indígenas brasileiros.

Método. Foi realizada uma revisão sistemática da literatura a partir das bases de dados PubMed, SciELO, PsycINFO e LILACS. Foram incluídos estudos de base populacional que enfocassem suicídio em populações indígenas no território brasileiro.

Resultados. A busca identificou 111 artigos, dos quais nove preencheram os critérios de inclusão. Três estudos foram realizados na região Centro-Oeste, quatro na região Norte do Brasil e dois abordaram todas as regiões do Brasil. Três estudos citaram as etnias estudadas, totalizando sete etnias (Terena, Kadiweu, Guato, Ofaie-Xavante, Guarani, Guarani-Kaiowá e Guarani-Nandeva). Os estudos demonstraram maior taxa de mortalidade por suicídio em pessoas do sexo masculino, solteiros, com 4 a 11 anos de escolaridade, na faixa etária de 15 a 24 anos, no domicílio e nos finais de semana, tendo como principal método o enforcamento. Os principais fatores de risco para o suicídio foram pobreza, fatores históricos e culturais, baixos indicadores de bem estar, desintegração das famílias, vulnerabilidade social e falta de sentido de vida e futuro.

Conclusões. Todos os estudos indicaram a necessidade de desenvolvimento de estratégias em conjunto com as comunidades, considerando sua cosmovisão e os aspectos sócio-histórico-culturais de cada etnia, para minimização dos fatores de risco e redução da taxa de suicídio.
\end{abstract}

Palavras-chave População indígena; suicídio; revisão sistemática; Brasil.

Os povos indígenas representam $0,4 \%$ da população total do Brasil. Caraterizados pela diversidade, apresentam configurações particulares de costumes, crenças e línguas (1). Em comum, constata-se que esses povos enfrentam constantemente situações de tensão social, com ameaça à integridade de seus territórios e saberes (2); essa insegurança os coloca em posição de maior vulnerabilidade frente a uma série de agravos (3) e problemas concretos, como invasões territoriais, exploração sexual (4) e uso abusivo de álcool $(5,6)$.

Conforme a Fundação Nacional de Saúde (Funasa) (2), a depressão e o suicídio são cada vez mais frequentes em diversas comunidades indígenas brasileiras. De fato, o Ministério da Saúde (7) do Brasil aponta que a população indígena brasileira apresenta altas taxas de suicídio, assim como relatado em populações nativas da Europa (8), América do Norte (9-11), Oceania $(12,13)$ e Ásia (14).

Na população geral do Brasil, o número de óbitos por suicídio cresceu 33,6\% de 2002 até 2012 (7). Com taxa geral variando de 5,3 suicídios por 100 mil habitantes em 2011 a 5,7 por 100 mil habitantes em 2015, foram registrados, nesse período, 55649 óbitos por suicídio no país. De 2008 a 2012, os suicídios indígenas representaram $1,0 \%$ dos óbitos registrados no

Universidade Federal de Minas Gerais (UFMG), Programa de Pós-Graduação em Psicologia, Belo Horizonte (MG), Brasil.

3 Universidade Federal de Minas Gerais (UFMG), Programa de Pós-Graduação em Medicamentos e Assistência Farmacêutica, Belo Horizonte (MG), Brasil. 
território brasileiro (15), um quantitativo 2,5 vezes maior do que a proporção de indígenas da população total do país. Em consonância com esse achado, dados da Secretaria Especial de Saúde Indígena (SESAI) (16) mostram que a taxa de suicídio em indígenas brasileiros no ano de 2014 foi de 21,8 por 100 mil habitantes - praticamente quatro vezes maior do que na população brasileira em geral.

Ressalta-se que, no contexto indígena, o suicídio está relacionado com fatores sociais e culturais específicos, que mudam radicalmente a epidemiologia e a etiologia do fenômeno (17). Portanto, o suicídio nas populações indígenas tem se mostrado um problema de saúde desafiador. Ao mesmo tempo, há poucos estudos epidemiológicos e antropológicos sobre o fenômeno no cenário nacional (18). Uma revisão sistemática do comportamento suicida indígena no mundo (19) identificou três estudos populacionais no Brasil; outra revisão com foco no suicídio indígena na América Latina (20) identificou poucos dados de estudos populacionais referentes ao Brasil. Diante do exposto, o presente estudo tem como objetivo descrever a frequência, as caraterísticas e os fatores que contribuem para o suicídio em povos indígenas brasileiros a partir de uma revisão sistemática da literatura.

\section{MATERIAIS E MÉTODOS}

Foi conduzida uma revisão sistemática de estudos que abordaram o suicídio em povos indígenas brasileiros. $\mathrm{O}$ artigo foi preparado de acordo com a ferramenta PRISMA, que auxilia na elaboração e análise de revisões sistemáticas (21). A questão de pesquisa (22) foi estruturada a partir da especificação de três componentes: população incluída nos estudos (povos indígenas brasileiros), delineamento dos estudos (estudos de base populacional) e desfecho analisado (óbitos registrados por suicídios).

Foi realizada uma busca eletrônica sistematizada de artigos publicados até fevereiro de 2020 nas bases de dados MEDLINE (PubMed), SciELO, PsycINFO e LILACS. Para cada base de dados, foi construída uma estratégia específica com descritores e sinônimos combinados de várias formas (tabela 1). Uma busca manual foi realizada nas referências bibliográficas dos estudos incluídos. Além disso, realizou-se uma busca na literatura cinzenta que incluiu a Biblioteca Digital Brasileira de Teses e Dissertações e o Banco de Teses da Capes. Não houve restrições quanto a data de publicação do estudo e idioma.

As publicações foram reunidas em uma única biblioteca, no programa EndNote, onde foram excluídas as duplicatas. Dois revisores (RSBS e JCO) realizaram a seleção dos estudos de forma independente, em duas fases: leitura de títulos e resumos; e leitura dos textos completos. As discordâncias foram resolvidas por um terceiro revisor (MLMT).

Foram incluídos estudos epidemiológicos observacionais e excluídos os estudos não populacionais, como relatos de caso, estudos com amostras de conveniência e estudos etnográficos. Ademais, os estudos selecionados também foram avaliados de acordo com os 22 critérios do STROBE (23), calculando a proporção dos critérios atendidos.

\section{RESULTADOS}

A revisão identificou 111 publicações. Após a avaliação em etapas, nove estudos foram incluídos segundo os critérios de elegibilidade estabelecidos (24-32). Os processos de seleção e
TABELA 1. Estratégia de busca da revisão sistemática sobre suicídio em povos indígenas brasileiros

\begin{tabular}{|c|c|c|}
\hline Base eletrônica & Estratégia de Busca & No. de estudos \\
\hline $\begin{array}{l}\text { MEDLINE (Pubmed) - } \\
\text { https://www.ncbi.nlm. } \\
\text { nih.gov/pubmed/ }\end{array}$ & $\begin{array}{l}\text { (((((()((((((Indigenous Population) } \\
\text { OR Indigenous Populations) } \\
\text { OR Population, Indigenous) } \\
\text { OR Populations, Indigenous) } \\
\text { OR Indigenous) OR Indigenous } \\
\text { communities) OR Natives) OR } \\
\text { Native-Born) OR Tribes))) AND } \\
\text { ((((((((((Suicide) OR Suicide, } \\
\text { Attempted) OR Suicide, Assisted) } \\
\text { OR Suicidal Ideation)) AND } \\
\text { ((((((((((((Population Groups) OR } \\
\text { Group, Population) OR Groups, } \\
\text { Population) OR Population } \\
\text { Group) OR Tribes) OR Natives) } \\
\text { OR Native-Born) OR Native Born) } \\
\text { OR Indigenous Population) OR } \\
\text { Indigenous Populations) OR } \\
\text { Population, Indigenous) OR } \\
\text { Populations, Indigenous)) AND } \\
\text { ((Brazil) OR Brazilian, Population))) } \\
\text { AND ((((((((Indigenous Population) } \\
\text { OR Indigenous Populations) } \\
\text { OR Population, Indigenous) OR } \\
\text { Populations, Indigenous) OR } \\
\text { Indigenous) OR Natives) OR Native- } \\
\text { Born) OR Tribes)) AND ((Brazil) } \\
\text { OR Brazilian, Population))) AND } \\
\text { (((((((((epidemiologic studies) OR } \\
\text { incidence) OR mortality) OR ecological } \\
\text { study) OR cohort) OR case control) OR } \\
\text { observational) OR population-based) } \\
\text { OR epidemiolog*)) AND ((Brazil) OR } \\
\text { Brazilian, Population) }\end{array}$ & 43 \\
\hline $\begin{array}{l}\text {-ILACS - https://lilacs. } \\
\text { bvsalud.org/ }\end{array}$ & $\begin{array}{l}\text { ((Indigenous Population OR Population, } \\
\text { Indigenous OR Indigenous OR Natives } \\
\text { OR Native-Born OR Tribes) AND } \\
\text { (Suicide OR Suicide, Attempted OR } \\
\text { Suicide, Assisted OR Suicidal Ideation) } \\
\text { AND (Brazil OR Brazilian, Population) } \\
\text { AND (epidemiologic studies OR } \\
\text { incidence OR mortality OR ecological } \\
\text { study OR cohort OR case control OR } \\
\text { observational OR population-based OR } \\
\text { epidemiolog*)) }\end{array}$ & 20 \\
\hline $\begin{array}{l}\text { SciELO - https://scielo. } \\
\text { org/ }\end{array}$ & $\begin{array}{l}\text { (Indigenous) AND (suicide) AND } \\
\text { (brazil) }\end{array}$ & 16 \\
\hline $\begin{array}{l}\text { SYCinfo - https:// } \\
\text { www.apa.org/pubs/ } \\
\text { databases/psycinfo/ }\end{array}$ & $\begin{array}{l}\text { Any Field: Indigenous AND suicide } \\
\text { AND brazil }\end{array}$ & 10 \\
\hline
\end{tabular}

exclusão estão descritos na figura 1. Na avaliação segundo o STROBE, todos os artigos atenderam percentuais acima de $60 \%$ dos critérios de qualidade (tabela 2).

Os estudos incluídos foram publicados de 2006 a 2019. A maioria $(24-29,31,32)$ utilizou como fonte de dados o Sistema de Informação de Mortalidade (SIM) do Ministério da Saúde. A tabela 3 resume as características dos estudos incluídos.

Três estudos $(24,25,30)$ abordaram o suicídio na região Centro-Oeste e quatro estudos (26-29) na região Norte. Dois estudos abordaram todas as regiões do Brasil $(31,32)$. Os estados mais abordados foram Mato Grosso do Sul $(24,25,30)$ e Amazonas (27-29). A maior taxa de suicídios de indígenas foi registrada no estado de Mato Grosso do Sul (25) (83,60/100 000 habitantes) e a menor, no Amazonas (27) (4,20/100 000 habitantes); essas 
FIGURA 1. Fluxograma da seleção de estudos para a revisão sistemática sobre suicídio em povos indígenas brasileiros

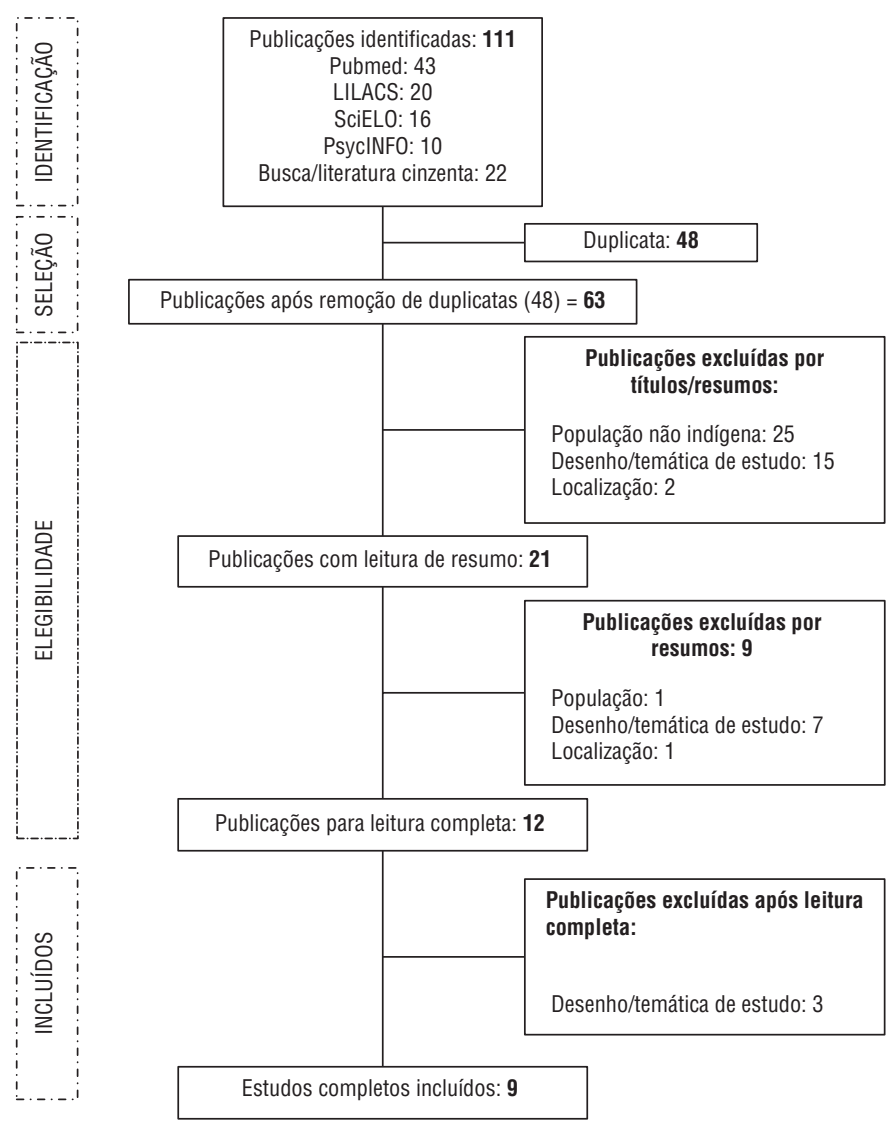

taxas foram medidas em diferentes intervalos temporais no período de 2005 a 2012. Somente três estudos $(24,25,30)$ citaram as etnias estudadas, sendo elas Terena, Kadiweu, Guato, Ofaie-Xavante, Guarani, Guarani-Kaiowá e Guarani-Nandeva.

Os estudos encontrados (tabela 3) mostraram maior frequência de suicídio em pessoas do sexo masculino (24-26, 28-32), solteiros (27-29) e com 4 a 11 anos de escolaridade (28). Lazzarini et al. (24) destacam que a taxa de suicídio no sexo masculino $(107,5 / 100000)$ excedeu o dobro da taxa no sexo feminino $(41,7 / 100$ 000). No contexto do suicídio entre crianças indígenas, diferentemente do observado em outras faixas etárias, encontrou-se uma tendência de magnitude igual em ambos os sexos (32).

No estudo de Victal et al. (31), os suicídios foram mais frequentes na faixa de 15 a 24 anos no sexo masculino (135,8/100 000). A faixa etária mais acometida nesse estudo foi a de 15 a 19 anos (31). No sexo feminino, a maior taxa foi observada na faixa de 10 a 14 anos - 38,3/100 000 (25).

Acerca do local do óbito, o domicílio foi o local mais frequente (25-28, 32). Três estudos (25-27) ressaltaram a ausência de óbito por suicídio em contexto hospitalar nos municípios investigados. Foi apontado que as crianças indígenas morreram menos frequentemente por suicídio em ambiente hospitalar quando comparadas às não indígenas (32). $\mathrm{O}$ principal método de suicídio utilizado pelos indígenas foi o enforcamento (24-26, 28, $29,32)$. Os suicídios indígenas ocorreram com maior frequência nos finais de semana $(25,27-29)$.
TABELA 2. Pontuação e percentual de qualidade dos artigos a partir dos critérios do STROBE

\begin{tabular}{lcc}
\multicolumn{1}{c}{ Estudos } & Itens & $\%$ \\
\hline Souza e Orellana (28) & 21 & 95,45 \\
Lazzarini et al. (24) & 20 & 90,91 \\
Orellana et al. (25) & 20 & 90,91 \\
Souza (32) & 19 & 86,36 \\
Souza e Junior (26) & 18 & 81,82 \\
Orellana et al. (27) & 18 & 81,82 \\
Souza e Orellana (29) & 17 & 77,27 \\
Victal et al. (31) & 17 & 77,27 \\
Coloma et al. (30) & 14 & 63,64 \\
\hline
\end{tabular}

a A lista de verificação Strengthening the Reporting of Observational Studies in Epidemiology (STROBE) é composta de 14 itens, estratificados ou não em subitens, totalizando 22 itens. Cada item recebeu uma pontuação proporcional, com somatória máxima de 22 pontos. Para este estudo, foram incluídos artigos que atenderam os itens STROBE com pontuação mínima de $60 \%$.

Os estudos associaram os dados encontrados com fatores de risco (tabela 4) identificados em estudos antropológicos e de observação-participante. Pobreza, fatores históricos e culturais, baixos indicadores de bem estar, desintegração das famílias (24), vulnerabilidade social (28), falta de sentido de vida e futuro (30), escassez de terras, dificuldades no gerenciamento de conflitos entre gerações, afastamento dos jovens das atividades tradicionais de subsistência e ter um familiar que morreu por suicídio (32) foram apontados como motivadores para as altas taxas de suicídio nos povos indígenas. Cinco estudos (25, $28,29,31,32)$ demonstraram que o álcool pode estar relacionado aos suicídios nas aldeias. Em nenhum estudo foi citada a presença de transtornos mentais associados aos comportamentos suicidas nos indígenas.

Algumas propostas de intervenção foram sugeridas nos estudos para estimular profissionais a compreenderem e evitarem esse comportamento na população indígena. Lazzarini et al. (24) sugerem ações na escola, visando a instrumentalizar crianças e jovens para enfrentar emoções negativas e comportamentos de risco de vida. Os autores (24) também enfatizam o uso de estratégias locais e o desenvolvimento da capacidade de comunicar sentimentos relacionados ao suicídio para os professores e familiares. Na área da saúde, sugere-se a realização de triagens para identificação de fatores de risco, além de acolhimento e intervenção aos enlutados para melhor entendimento do ato suicida e prevenção para contenção futura (28). No âmbito do suicídio de crianças indígenas, foi destacada a necessidade de prevenção da violência e de agravos, com ações como o combate ao bullying nas escolas e a postergação da idade do início do consumo de álcool e outras drogas (32).

Duas pesquisas $(27,30)$ destacaram a importância da realização de novas investigações acerca do fenômeno de suicídio indígena, com participação dos próprios indígenas (27) na construção conjunta de estratégias de enfrentamento culturalmente sensíveis ao cenário sociocultural e sanitário (29). Souza e Onety Júnior (26) propõem a realização de estudos com o objetivo de investigar o processo de urbanização da população e seus impactos nas comunidades. Orellana et al. (25) pontuam a necessidade de fortalecer as políticas indigenistas para proteção das comunidades, evitando novas violações de direitos. 
TABELA 3. Características dos estudos incluídos na revisão sistemáticaa

\begin{tabular}{|c|c|c|c|c|c|c|c|}
\hline Estudo & Região & Etnia & $\begin{array}{l}N^{0} \text { de mortes por } \\
\text { suicídio }\end{array}$ & $\begin{array}{l}\text { Intervalo de tempo } \\
\text { estudado }\end{array}$ & $\begin{array}{l}\text { Base de } \\
\text { dados }\end{array}$ & $\begin{array}{c}\text { Taxa de suicídio } \\
\text { (óbitos/ } 100000 \\
\text { pessoas) }\end{array}$ & $\begin{array}{l}\text { Desenho do } \\
\text { estudo }\end{array}$ \\
\hline Lazzarini et al. (24) & Mato Grosso do Sul & $\begin{array}{l}\text { Guarani-Kaiowá, } \\
\text { Terena e Guarani }\end{array}$ & 119 & 2003-2013 & SIM & 73,4 & $\begin{array}{l}\text { Coorte } \\
\text { retrospectivo }\end{array}$ \\
\hline Orellana et al. (25) & Mato Grosso do Sul & $\begin{array}{l}\text { Guarani-Kaiowá, } \\
\text { Terena e } \\
\text { Guarani-Nandeva }\end{array}$ & 118 & $2000-2012$ & SIM & 83,6 & $\begin{array}{l}\text { Ecológico } \\
\text { descritivo } \\
\text { com foco no } \\
\text { espaço-temporal }\end{array}$ \\
\hline Orellana et al. (27) & $\begin{array}{l}\text { Amazonas (SGC, SIRN } \\
\text { e TBT) }\end{array}$ & NE & 113 & 2005-2009 & SIM & 4,2 & $\begin{array}{l}\text { Descritivo e } \\
\text { retrospectivo }\end{array}$ \\
\hline Souza e Orellana (28) & Amazonas & NE & 131 & $2006-2010$ & SIM & 18,4 & $\begin{array}{l}\text { Coorte } \\
\text { retrospectivo }\end{array}$ \\
\hline Souza e Orellana (29) & $\begin{array}{l}\text { São Gabriel da } \\
\text { Cachoeira (AM) }\end{array}$ & NE & 44 & $2000-2007$ & SIM & 16,8 & $\begin{array}{l}\text { Descritivo } \\
\text { retrospectivo } \\
\text { quantitativo }\end{array}$ \\
\hline Victal et al. (31) & Brasil & $\mathrm{NE}$ & 935 & 2005-2014 & SIM & 10,50 & $\begin{array}{l}\text { Epidemiológico } \\
\text { transversal }\end{array}$ \\
\hline Souza (32) & Brasil & NE & 55 & $2010-2014$ & SIM & 11,0 & Descritivo \\
\hline
\end{tabular}

${ }^{a}$ NE: não explicitado; SIM: Sistema de Informações sobre Mortalidade; SGC: São Gabriel da Cachoeira; SIRN: Santa Isabel do Rio Negro; TBT: Tabatinga.

TABELA 4. Fatores de risco para suicídio na população indígena brasileira apontados na revisão de literatura

\begin{tabular}{lc}
\multicolumn{1}{c}{ Fatores de Risco } & Estudos \\
Abandono das tradições indígenas & $24,25,27,28,29,31,32$ \\
Consumo abusivo de álcool e/ ou abuso de & $24,25,27,28,29,31,32$ \\
drogas & $24,26,27,29$ \\
Falta de acesso a educação e trabalho & $24,29,32$ \\
Vulnerabilidade socioeconômica & $25,28,30$ \\
Instabilidade emocional dos jovens & $25,30,31,32$ \\
Confinamento territorial e reassentamentos & 24,25 \\
Violência sexual & 26,30 \\
Aproximação da população urbana & $29,23,32$ \\
Fragilização cultural & 26 \\
Exposição a meios letais & 30 \\
Imposição religiosa e cultural & 32 \\
Ter um familiar que morreu por suicídio & 24 \\
Casa como local para planos suicidas &
\end{tabular}

\section{DISCUSSÃO}

O suicídio, compreendido como um ato deliberado executado pelo próprio indivíduo, cuja intenção seja a morte, de forma consciente e intencional, mesmo que ambivalente, usando um meio que se acredita ser letal (33), é um fenômeno complexo no contexto indígena, sendo necessário discutir alguns aspectos descritos e analisados. Um desses pontos diz respeito às limitações da fonte de dados mais utilizada nos estudos (24-29), o SIM, sistema de vigilância epidemiológica nacional que fornece informações acerca da mortalidade para todas as instâncias do sistema de saúde brasileiro.
As limitações do uso do SIM decorrem da incompletude no preenchimento dos formulários e de possíveis problemas durante a transferência das informações dos formulários, por parte do profissional de saúde, para o sistema e posterior construção do banco de dados; também decorrem de problemas na cobertura dos óbitos, principalmente em municípios rurais e remotos, e da subnotificação de suicídios por parte de profissionais de saúde $(25,29,31,32)$. Este último ponto pode estar relacionado com a heteroclassificação da raça na declaração do óbito, ou seja, a identificação dos indivíduos enquanto indígenas por observação externa dos profissionais de saúde, processo atravessado por preconceitos e dificuldades também decorrentes da identificação pelos familiares (27). Tais limitações fazem com que os dados secundários sejam muitas vezes classificados como de pouca qualidade (27). Ressalta-se, nesse contexto, que a SESAI tem apostado na ampliação e qualificação das informações acerca do suicídio indígena, considerando que a vigilância epidemiológica contribui para a compreensão desse fenômeno e, consequentemente, possibilita o planejamento de estratégias para a sua prevenção (34).

As regiões mais abordadas nos estudos, Centro-Oeste e Norte, são as que concentram as maiores taxas de suicídio do Brasil, sendo que, no Centro-Oeste, a taxa de suicídio indígena é 7 vezes maior do que na população não indígena; e na região Norte, a taxa é 5 vezes maior do que na população não indígena (29). Os estudos mostram que essas são as áreas com as maiores taxas de suicídio indígena $(31,32)$, corroborando os achados de Weizelfisz (15). Em consonância, a maior taxa de suicídio foi identificada no estado de Mato Grosso do Sul (25), no Centro-Oeste; entretanto, a menor taxa foi encontrada no estado do Amazonas, na região Norte (27). Uma explicação para esse achado é que o estudo foi realizado no estado do Amazonas em municípios com alta proporção de população autodeclarada 
indígena, porém também incluiu o município de Manaus, que apresenta baixa representatividade de autodeclarados indígenas. De fato, embora a taxa de mortalidade tenha sido baixa, a análise comparativa entre municípios indicou diferenças no padrão de mortalidade, com destaque para as elevadas taxas nos municípios que possuíam alta proporção de população autodeclarada indígena, bem como grande frequência de óbitos entre indivíduos indígenas (27).

O Norte e o Centro-Oeste são as regiões que possuem o maior número de etnias declaradas e o maior número de terras indígenas no país (1). Embora esses fatores não justifiquem as altas taxas de suicídio, podem contribuir para compreendê-las, uma vez que um número maior de indígenas em determinada região implica, no histórico do país, conflitos com pessoas não indígenas, principalmente relacionadas a demarcações de terras. Em consonância, um estudo considerou como possível explicação as mudanças ocorridas nas terras indígenas demarcadas no Amazonas, com aumento da presença de não indígenas e da urbanização da população indígena, juntamente com aumento de fatores de risco como maior consumo de álcool e conflitos entre gerações (32). Argumenta-se que, no arco do desmatamento amazônico, o alto índice de violência pode estar relacionado a interesses políticos e econômicos em torno de empreendimentos agrícolas (15). Em Mato Grosso do Sul, a explicação também pode envolver tensões com o agronegócio, bem como a escassez de terras indígenas demarcadas (32).

Nos estudos analisados, apenas sete etnias foram identificadas. Ressalta-se que os indígenas brasileiros são pertencentes a 305 diferentes etnias, com ampla diversidade entre si (1), e que cada povo indígena possui sua própria forma de entender e significar o suicídio (34). Assim, a falta de identificação das etnias nos estudos pode ser um limitador da compreensão do suicídio indígena brasileiro, uma vez que esse fenômeno possui peculiaridades específicas a cada etnia (31).

A identificação de maior mortalidade por suicídio nos indígenas de sexo masculino (24-26, 28-30) corrobora a revisão sistemática realizada por Pollock et al. (19). Essa revisão anterior mostrou que a maioria das mortes por suicídio ocorreu em homens em todos os estudos analisados. Na população geral do Brasil, o risco de suicídio no sexo masculino é aproximadamente 4 vezes maior do que no sexo feminino, embora o risco tenha aumentado, ao longo do tempo, para ambos os sexos (7).

Estudos descreveram os locais de óbito, havendo destaque para a ausência de óbitos por suicídio em contexto hospitalar (25-37). Na população geral, a ocorrência de suicídios em hospitais é alta (35). Tal discrepância pode estar relacionada com o fato de que os povos indígenas têm seu acesso aos serviços de saúde restrito (32), ainda que a implementação da Política Nacional de Atenção à Saúde dos Povos Indígenas tenha buscado superar as deficiências de cobertura, acesso e aceitabilidade do SUS para essa população (3).

No âmbito de tal discussão, na população geral mais de $90 \%$ dos casos de suicídio avaliados tinham a presença de sintomas que preencheriam critérios para o diagnóstico de transtorno mental (36). A ausência do mapeamento de possíveis transtornos mentais associados aos comportamentos suicidas nos estudos com indígenas pode ser uma lacuna na compreensão e prevenção desse fenômeno. Ao mesmo tempo, deve-se considerar a complexidade da transposição de conceitos do universo ocidental para o indígena (32). Ainda, um estudo acerca da saúde mental dos povos indígenas deve ser, necessariamente, multidisciplinar, não cabendo apenas à psiquiatria e à psicologia o protagonismo na discussão dos fenômenos em questão (37).

Por sua vez, o álcool foi citado como presente nos contextos de suicídio indígena $(25,28,29,31,32)$. Conforme a Política Nacional de Saúde dos Povos Indígenas (2), o alcoolismo e sua relação com a violência aparecem entre as sete situações especiais a serem prioritariamente enfrentadas, dada a sua complexidade e relevância sanitária. A gravidade e a urgência de solucionar esse problema são ratificadas pela Política de Atenção à Saúde Mental das Populações Indígenas (38).

Considerando que, no contexto indígena, há "uma complexa conjuntura social que compõe o quadro de fatores propiciadores da consumação do ato" (16; p. 6), os estudos apontaram fatores externos para explicar os óbitos por suicídio, com destaque para o reassentamento e realocação das terras $(25,32)$. Segundo Machado e Santos (39), a falta de pertencimento a um território pode afetar as comunidades indígenas, pois o espaço físico tem um papel político e social fundamental na sua organização, e cada vez mais esses grupos sofrem os impactos da falta de demarcação dos seus territórios e das desapropriações de suas terras. A demarcação de terras, porém, "não esgota os desafios de sobrevivência dos povos indígenas", que vivenciam relações de subalternização com o estado brasileiro (32; p.7).

De acordo com Bom Meihy (40), não é suficiente compreender o suicídio indígena a partir de fatores "de fora", já que esses fatores não contemplam a mais importante das explicações, que é a visão da população indígena sobre a morte. Essa compreensão restrita pode submeter, incorporar ou anular o papel de sujeito da população indígena, desconsiderando sua cosmovisão e representação do universo simbólico da comunidade a partir de crenças e valores construídos ao longo da história (40). As singularidades e cosmovisões, em uma dimensão singular, devem, pois, ser consideradas, sendo necessário pesquisar como os próprios indígenas dão sentido ao fenômeno do suicídio (32).

Ao mesmo tempo, deve-se entender quais condições objetivas e sociais que comprometem o modo de vida tradicional impõem limitações para o bem viver indígena, podendo fazer prevalecer o sofrimento, inclusive com impulso ao suicídio (41). O líder indígena Antonísio Lulu Darã (42) referiu-se a uma região na qual estavam acontecendo muitos suicídios pela ausência de terra, rios, moradia e material para o artesanato, questionando: "para os nossos antepassados, a natureza era os nossos conselheiros e psicólogos, porque quando o índio está dentro da aldeia ele pega a flecha, pega o arco dele vai para dentro da mata e lá ele vai refletir. E hoje, como fazer isso?" (42).

Os estudos analisados nesta revisão apresentam algumas propostas de enfrentamento que podem contribuir para a prevenção do suicídio, uma vez que esta é uma morte evitável e, diferente de outros agravos, existem ferramentas para sua contenção (43). Entre os fatores de proteção e prevenção do suicídio preconizados mundialmente, a Organização Mundial da Saúde (OMS) (44) destaca as iniciativas de suporte psicossocial para a comunidade, sobretudo para famílias e grupos com histórico de tentativas de suicídio e abuso de álcool e outras drogas. Tais ações devem contemplar triagem, acolhimento, monitoramento e avaliação nos territórios, respeitando as diferenças culturais de cada povo, valorizando os saberes tradicionais levando em conta também o conhecimento científico (45).

Acerca da produção de novos estudos $(27,30)$, é recomendável no futuro a utilização de métodos combinados, de mais 
pesquisas epidemiológicas e do uso de instrumentos como a entrevista de autópsia psicológica, além da realização de estudos etnográficos que dialoguem com diferentes áreas do conhecimento. Para conhecer melhor o comportamento suicida em comunidades indígenas, faz-se necessário o uso de delineamentos quali-quantitativos $(32,34,46)$, que auxiliam na compreensão do fenômeno, tanto individual quanto socialmente.

Em conclusão, sumarizar os estudos (24-32) que retratam, por meio de delineamento populacional, o cenário nacional do suicídio nos povos indígenas brasileiros permitiu ampliar a compreensão desse complexo problema de saúde pública. Os estudos demonstraram maior incidência de suicídio em pessoas do sexo masculino, solteiras, com 4 a 11 anos de escolaridade, na faixa etária de 15 a 24 anos, no domicílio e nos finais de semana, tendo como principal método o enforcamento. Os principais fatores de risco para o suicídio foram pobreza, desvantagem socioeconômica, fatores históricos e culturais, baixos indicadores de bem estar, desintegração das famílias, vulnerabilidade social e falta de sentido de vida e futuro.

Tais achados podem contribuir para a construção de uma política de saúde que vise à prevenção do suicídio indígena, além de impulsionar ações que busquem a garantia dos direitos das populações indígenas e de intervenções com foco na população não indígena para o combate do preconceito e demais violências. Sugere-se investimento em pesquisas que englobem um número maior de etnias para a compreensão do suicídio aliado à cosmovisão dessas populações, bem como a realização de estudos por meio dos sistemas de informação em saúde utilizando critérios claros e válidos para essas populações. A parceria entre comunidades indígenas, serviços de saúde e universidades pode representar um caminho assertivo para a construção de tais ações de pesquisa e intervenção.

Contribuição dos autores. RSBS e MLMT conceberam a ideia original. RSBS, JCO e MLMT coletaram e analisaram os dados. RSBS, JCO, JA-T e MLMT escreveram o artigo. Todos os autores revisaram e aprovaram a versão final.

\section{Conflitos de interesse. Nada declarado pelos autores.}

Financiamento. Maycoln Leôni Martins Teodoro possui bolsa de produtividade 1D do Conselho Nacional de Desenvolvimento Científico e Tecnológico (CNPq).

Declaração. As opiniões expressas no manuscrito são de responsabilidade exclusiva dos autores e não refletem necessariamente a opinião ou política da RPSP/PAJPH ou da Organização Pan-Americana da Saúde (OPAS).

\section{REFERÊNCIAS}

1. Instituto Brasileiro de Geografia e Estatística. Características Gerais dos Indígenas - Resultado do Universo": Censo Demográfico. Rio de Janeiro: IBGE. 2010. Disponível em: https://biblioteca.ibge.gov. $\mathrm{br} /$ index.php $/$ biblioteca-catalogo $\mathrm{id}=795 \& \mathrm{view}=$ detalhes. Acesso em 17 de outubro de 2019.

2. Fundação Nacional de Saúde. Política nacional de atenção à saúde dos povos indígenas. $2^{\mathrm{a}}$ ed. Brasília: Fundação Nacional de Saúde/ Ministério da Saúde. 2002. Disponível em: http:/ /bvsms.saude.gov. $\mathrm{br} /$ bvs/publicacoes/politica_saude_indigena.pdf. Acesso em 17 de outubro de 2019.

3. Coimbra Jr. CEA, Santos RV. Minorias e desigualdades: algumas teias de inter-relações, com ênfase nos povos indígenas no Brasil. Ciênc Saúde Coletiva. 2000; 5(1):125-32. https://dx.doi.org/10.1590/S1413-81232000000100011. Disponível em: http://www.scielo.br/scielo.php?script=sci_abstract\&pid=S1413-81232000000100011\&lng=pt\&nrm=iso\&tlng=pt. Acesso em 17 de outubro de 2019.

4. Paiva MVS. Cultura indígena e identidade nacional. Revista Temas em Educação e Saúde, Araraquara. 2016, 12(2):230-245.

5. Souza MLP. Processos de alcoolização indígena no Brasil: Perspectivas plurais. Rio de Janeiro, RJ: Fiocruz; 2013.

6. Souza RSB, Oliveira JC, Teodoro MLM. Construção de um Instrumento para Avaliar o Uso de Bebidas Alcóolicas em uma Etnia Indígena de Minas Gerais. Psicologia: Ciência e Profissão. 2019; 39: 1-11. https://dx.doi.org/10.1590/1982-3703003176628. Disponível em: http:/ / www.scielo.br/scielo.php?script=sci_arttext\&pid=S1414-98932019000100104\&lng=pt\&tlng=pt. Acesso em 17 de outubro de 2019.

7. Ministério da Saúde. Secretaria de Vigilância em Saúde. Suicídio. Saber, agir e prevenir. Perfil epidemiológico das tentativas e óbitos por suicídio no Brasil e a rede de atenção à saúde. Boletim Epidemiológico. 2017;48(30). Disponível em: http://portalarquivos. saude.gov.br/images/pdf/2017/setembro/21/2017-025-Perfil-epidemiologico-das-tentativas-e-obitos-por-suicidio-no-Brasil-e-a-rede-de-atencao-a-saude.pdf. Acesso em 17 de outubro de 2019.

8. Silviken A. Prevalence of suicidal behavior among indigenous Sami in northern Norway. Int J Circumpolar Health.
2009,68:(3):204-11. Disponível em: https:/ /www.ncbi.nlm.nih.gov / pubmed/19705653. Acesso em 17 de outubro de 2019.

9. Mullany B, Barlow A, Goklish N, Larzelere-Hinton F, Cwik M, Craig $\mathrm{M}$, et al. Toward understanding suicide among youths: results from the White Mountain Apache tribally mandated suicide surveillance system, 2001-2006. Am J Public Health. 2009;99:1840-8. Disponível em: https://www.ncbi.nlm.nih.gov/pmc/articles/PMC2741522/. Acesso em 17 de outubro de 2019.

10. Kirmayer LJ, Brass GM, Holton T, Paul K, Simpson C, Tait C. Suicide among aboriginal people in Canada. Ottawa: The Aboriginal Healing Foundation; 2007. Disponível em: http:/ /www.douglas.qc.ca/ uploads/File/2007-AHF-suicide.pdf. Acesso em 17 de outubro de 2019.

11. Mullany B, Barlow A, Goklish N, Larzelere-Hinton F, Cwik M, Craig M, Walku, J,T.p. Toward Understanding Suicide Among Youths: Results From the White Mountain Apache Tribally Mandated Suicide Surveillance System, 2001-2006. Am J Public Health. 2010;99(10):1840-8. Disponível em: https:/ / www.ncbi.nlm.nih.gov/ pmc/articles/PMC2741522/. Acesso em 17 de outubro de 2019.

12. Kuipers $\mathrm{P}$, Appleton J, Pridmore S. Thematic analysis of key factors associated with Indigenous and non-Indigenous suicide in the Northern Territory, Australia. Rural Remote Health. 2012;12:2235. Disponível em: https://core.ac.uk/download/pdf/143865848.pdf. Acesso em 17 de outubro de 2019

13. Silburn S, Glaskin B, Henry D, Drew N. Preventing Suicide Among Indigenous Australians. En: Purdie N, Dudgeon P. \& Walker R, (eds). Working Together: Aboriginal and Torres Strait Islander Mental Health and Wellbeing Principles and Practice. pp. 91-104 Canberra: Office of Aboriginal and Torres Strait Islander Health, Department of Ageing; 2010.

14. Sumarokov YA, Brenn T, Kudryavtsev AV, Nilssen O. Suicides in the indigenous and non-indigenous populations in the Nenets Autonomous Okrug, Northwestern Russia, and associated socio-demographic characteristics. Int J Circumpolar Health. 2014;6(73):24308. doi: 10.3402/ijch.v73.24308. eCollection 2014. Disponível em: https://www.ncbi.nlm.nih.gov/pubmed/25006556. Acesso em 17 de outubro de 2019. 
15. Weizelfisz JJ. Os jovens do Brasil: mapa da violência. Rio de Janeiro: FLACSO; 2014. Disponível em: https:/ / www.mapadaviolencia.org. br/mapa2014_jovens.php. Acesso em 17 de outubro de 2019.

16. Ministério da Saúde. Secretaria especial de saúde indígena. Agenda estratégica de ações de prevenção do suicídio em populações indígenas 2017 - 2018. Brasil: Ministério da Saúde; 2017. Disponível em: http://portalarquivos.saude.gov.br/images/pdf/2017/setembro/21/Coletiva-suicidio-21-09.pdf. Acesso em 17 de outubro de 2019.

17. Ministério da Saúde. Secretaria Especial de Saúde Indígena. Departamento de Atenção à Saúde Indígena. Coordenação-Geral de Atenção Primária “a Saúde Indígena. Área técnica de Saúde Mental e Medicinas Tradicionais- CGAPSI/DASI/SESAI. Material Orientador para Prevenção do Suicídio em Povos Indígenas. Brasília: Ministério da Saúde; 2015.

18. Souza MLP, Ferreira LO. Jurupari se suicidou?: notas para investigação do suicídio no contexto indígena. Saúde e Sociedade. 2014;23(3):1064-76. Disponível em: http://www.scielo.br/scielo. php?pid=S0104-12902014000301064\&script=sci_abstract\&tlng=pt. Acesso em 17 de outubro de 2019.

19. Pollock NJ, Naicker K, Loro A, Mulay S, Colman I. Global incidence of suicide among Indigenous peoples: a systematic review. BMC Med. 2018;16(1):145. doi: 10.1186/s12916-018-1115-6.

20. Azuero AJ, Arreaza-Kaufman D, Coriat J, Tassinari S, Faria A, Castañeda-Cardona C, Rosselli D. Suicide in the Indigenous Population of Latin America: A Systematic Review. Rev Colomb Psiquiatr. 2017;6(4):237-42. doi: 10.1016/j.rcp.2016.12.002.

21. Moher D, Liberati A, Tetzlaff J, Altman DG, Group P. Preferred reporting items for systematic reviews and meta-analyses: the PRISMA statement. PLoS. 2009; 6(7):2-9. https://doi.org/10.1371/ journal.pmed.1000097

22. Ministério da Saúde. Secretaria de Ciência, Tecnologia e Insumos Estratégicos. Departamento de Ciência e Tecnologia. Diretrizes metodológicas: elaboração de revisão sistemática e metanálise de ensaios clínicos randomizados/ Ministério da Saúde, Secretaria de Ciência, Tecnologia e Insumos Estratégicos, Departamento de Ciência e Tecnologia. Brasília: Editora do Ministério da Saúde; 2012.

23. von Elm E, Altman DG, Egger M, Pocock SJ, Gotzsche PC, Vandenbroucke JP. The Strengthening the Reporting of Observational Studies in Epidemiology (STROBE) statement: guidelines for reporting observational studies. J Clin Epidemiol. 2008; 61:344-9.

24. Lazzarini T, Gonçalves C, Benites W, Silva L, Tsuha D, Ko A, Rohrbaugh R, Andrews J, Croda J. Suicide in Brazilian indigenous communities: clustering of cases in children and adolescents by household. Rev Saude Publica. 2018;52:56. https://doi.org/10.11606/ S1518-8787.2018052000541. Disponível em: http://www.scielo. br/scielo.php?script=sci_arttext\&pid=S0034-89102018000100253. Acesso em 17 de outubro de 2019.

25. Orellana JD, Balieiro AA, Fonseca FR, Basta PC, Souza MLP. Spatial-temporal trends and risk of suicide in Central Brazil: an ecological study contrasting indigenous and non-indigenous populations. Br J Psychiatry. 2016;38(3):222-230. https://dx.doi. org/10.1590/1516-4446-2015-1720

26. Souza MLP de, Onety Júnior RT da S. Caracterização da mortalidade por suicídio entre indígenas e não indígenas em Roraima, Brasil, 2009-2013. Epidemiol Serv Saude. 2017;26(4):887-893. https://dx. doi.org/10.5123/s1679-49742017000400019.

27. Orellana JDY, Basta PC, Souza MLP de. Mortalidade por Suicídio: um enfoque em municípios com alta proporção de população autodeclarada indígena no Estado do Amazonas, Brasil. Rev Bras Epidemiol. 2013;16(3):658-69. https://dx.doi.org/10.1590/ S1415-790X2013000300010.

28. Souza MLP, Orellana JDY. Desigualdades na mortalidade por suicídio entre indígenas e não indígenas no estado do Amazonas, Brasil. J Bras Psiquiatr. 2013; 62(4):245-252. https://dx.doi.org/10.1590/ S0047-20852013000400001

29. Souza MLP, Orellana JDY. Suicide among the indigenous people in Brazil: a hidden public health issue. Rev Bras Psiquiatr. 2012;34:489-492. Disponível em: http://www.scielo.br/scielo. php?script=sci_arttext\&pid=S1516-44462012000400017. Acesso em 17 de outubro de 2019.

30. Coloma CJ, Hoffman J, Crosby A. Suicide Among the Guarani Kaiowá and Nandeva in Mato Grosso do Sul, Brazil. Arch Suicide Res. 2006;10(2):191. http://dx.doi.org/10.1590/
S1414-98932011000300006. Disponível em: http://www.scielo. br/scielo.php?script=sci_arttext\&pid=S1414-98932011000300006. Acesso em 17 de outubro de 2019.

31. Victal VVJRC, Aguiar BT, Xavier Júnior AFS, Cabral Júnior CR. Suicídio e povos indígenas no Brasil. Interfaces Científicas - Saúde e Ambiente, Aracaju. 2019;7(3):49-60. doi: 10.17564/2316-3798.2019v7. Disponível em: https://periodicos.set.edu.br/index.php/saude/ article/view/6213/pdf. Acesso em 17 fevereiro de 2020.

32. Souza MLP. Mortalidade por suicídio entre crianças indígenas no Brasil. Cad. Saúde Pública. 2019;35(Sup 3):e00019219. Disponível em: http://www.scielo.br/scielo.php?script=sci_arttext\&pid=S0102-311X2019001505007\&lng=pt\&nrm=iso\&tlng=pt. Acesso em 17 fevereiro de 2020

33. Associação Brasileira de Psiquiatria. Suicídio: informando para prevenir. Associação Brasileira de Psiquiatria, Comissão de Estudos e Prevenção de Suicídio. - Brasília: CFM/ABP, 2014. Disponível em: https://www.abp.org.br/cartilha-combate-suicidio. Acesso em 01 de abril de 2020.

34. Ministério da Saúde. Secretaria Especial de Saúde Indígena. Departamento de Atenção à Saúde Indígena. Manual de Investigação/ Notificação de Tentativas e Óbitos por Suicídio em Povos Indígenas / Ministério da Saúde, Secretaria Especial de Saúde Indígena, Departamento de Atenção à Saúde Indígena. Brasília: Ministério da Saúde; 2019

35. Botega N. Comportamento suicida: epidemiologia. Psicologia USP. 2014;25(3): 231-236. https://doi.org/10.1590/0103-6564D20140004 Disponível em: http://www.scielo.br/scielo.php?pid=S0103-65642014000300231\&script=sci_abstract\&tlng=pt. Acesso em 17 de outubro de 2019

36. Bertolote JM, Fleischmann A. Suicide and psychiatric diagnosis: A worldwide perspective. World Psychiatry. 2002;1:181-5

37. Vianna JJB, Cedaro JJ. e Ott AMT. Aspectos psicológicos na utilização de bebidas alcoólicas entre os Karitiana. Psicologia \& Sociedade. 2012;24(1):94-103. http://dx.doi.org/10.1590/S010271822012000100011. Disponível em: http://www.scielo.br/scielo. php?pid=S0102-71822012000100011\&script=sci_abstract\&tlng=pt. Acesso em 17 de outubro de 2019.

38. Ministério da Saúde. Portaria n ${ }^{\circ} 2.759$ (25, outubro, 2007). Estabelece diretrizes gerais para a Política de Atenção Integral à Saúde Mental das Populações Indígenas e cria o comitê Gestor Brasil. Brasília: Ministério da Saúde; 2007.

39. Machado DB, Santos DN. Suicídio no Brasil, de 2000 a 2012. J Bras Psiquiat. 2015;64(1):45-54. https://dx.doi. org/10.1590/0047-2085000000056

40. Bom Meihy JCS. A morte como apelo para a vida: o suicídio. Em Kaiowá, em RV Santos \& CE Coimbra Jr. (orgs.) Saúde e povos indígenas ( pp. 243-251). Rio de Janeiro: Fiocruz; 1994.

41. Teixeira LCA. Psicologia na promoção do bem viver indígena. Em: Conselho Regional de Psicologia da $6^{a}$ Região (Org.). Povos indígenas e psicologia: a procura do bem viver. São Paulo: CRP SP; 2016.

42. Darã AL. Manifestações de lideranças indígenas. Conselho Regional de Psicologia da $6^{a}$ Região (Org.). Psicologia e povos indígenas. São Paulo: CRPSP; 2010.

43. Organização Mundial da Saúde. Ação de saúde pública para a prevenção de suicídio: uma estrutura. Departamento de Saúde Mental. OMS: Genebra; 2012.

44. Organização Mundial da Saúde. Prevenção do Suicídio: um recurso para conselheiros. OMS: Genebra; 2006.

45. Souza MLP. Narrativas indígenas sobre suicídio no Alto Rio Negro, Brasil: tecendo sentidos. Saude Soc. 2016;25(1):145-59. Disponível em: http://www.scielo.br/pdf/sausoc/v25n1/ 1984-0470-sausoc-25-01-00145.pdf. Acesso em 17 de outubro de 2019.

46. Hoifodt TS, Talseth AG, Olstad RA. Qualitative study of the learning process in young treating suicidal patients: from insecurity to personal pattern knowledge and self-confidence. BMC Med Educ. 2007;7:21. Disponível em: https://www.ncbi.nlm.nih.gov/pubmed/17612413. Acesso em 17 de outubro de 2019.

Manuscrito recebido em 28 de outubro de 2019. Aceito em versão revisada em 28 de fevereiro de 2020. 


\section{Suicide and indigenous populations in Brazil: systematic review}

ABSTRACT Objective. To describe the frequency, characteristics, and factors contributing to suicide in indigenous populations in Brazil.

Method. A systematic review of the literature was performed in PubMed, SciELO, PsycINFO, and LILACS. All population-based studies focusing on suicide among indigenous populations in Brazil were included.

Results. The search identified 111 articles, of which nine met the inclusion criteria. Three of these studies were performed in the Midwest and four in the North of Brazil, while two covered all Brazilian regions. The ethnic groups investigated were specified in three studies (Terena, Kadiweu, Guato, Ofaie-Xavante, Guarani, Guarani-Kaiowá, and Guarani-Nandeva). Suicide rates were highest among males, single individuals, those with 4 to 11 years of schooling, and those aged 15 to 24 years. Suicides occurred most often in the home and on weekends, mostly by hanging. The main risk factors for suicide identified in the articles were poverty, historical and cultural factors, poor wellbeing indicators, family disintegration, social vulnerability, and lack of life or future perspective.

Conclusions. All the studies indicated the need to engage communities in developing strategies, considering their cosmovision and the social, historic, and cultural view of each ethnic group to minimize risk factors and reduce suicide rates.

Keywords Indigenous population; suicide; systematic review; Brazil.

\section{El suicidio y los pueblos indígenas brasileños: revisión sistemática}

RESUMEN Objetivo. Describir la frecuencia y las características del suicidio y sus factores contribuyentes en los pueblos indígenas brasileños.

Método. Se realizó una revisión sistemática de la bibliografía a partir de las bases de datos PubMed, SciELO, PsycINFO y LILACS. Se incluyeron estudios de base poblacional enfocados en el suicidio en las poblaciones indígenas en el territorio brasileño.

Resultados. En la búsqueda se encontraron 111 artículos, de los cuales nueve se ciñeron a los criterios de inclusión. Se realizaron tres estudios en la región centro oeste y cuatro en la región norte, además de otros dos que abarcaron todas las regiones de Brasil. En tres estudios se citaron todas las siete etnias estudiadas (Terena, Kadiweu, Guato, Ofaie-Xavante, Guaraní, Guaraní-Kaiowá y E-Nandeva). En los estudios se demostró una mayor tasa de mortalidad por suicidio en personas solteras del sexo masculino, con 4 a 11 años de escolaridad, en el grupo de 15 a 24 años de edad, en el domicilio y en los fines de semana, principalmente por el método de ahorcamiento. Los principales factores de riesgo de suicidio fueron la pobreza, factores históricos y culturales, el índice de bienestar bajo, la desintegración de las familias, la vulnerabilidad social, y la falta de sentido de la vida y del futuro.

Conclusiones. Todos los estudios indicaron la necesidad de formular estrategias junto con las comunidades, dentro del marco de su cosmovisión y de los aspectos sociales, históricos y culturales de cada etnia, con el fin de reducir al mínimo los factores de riesgo y la tasa de mortalidad por suicidio.

Palabras clave Población indígena; suicidio; revisión sistemática; Brasil. 